\title{
A testing time for doctors: counselling patients before an HIV test
}

\author{
Robert Bor, Riva Miller, Margaret Johnson
}

There have been considerable advances in the diagnosis, assessment, treatment, and prevention of HIV infection since AIDS was first described in 1981. Because of these advances HIV testing will inevitably become more widespread and a more routine part of clinical investigation and diagnosis. Up to now, however, doctors have often been deterred from testing patients for HIV. This may be due in part to the apparent mystique that surrounds pre-HIV test counselling.

\section{Groundwork for testing and counselling}

Deciding whether to test for HIV has always been difficult because social, psychological, and ethical consequences have tended to be as pressing as the medical implications. These have deterred patients from coming forward for testing and some doctors from suggesting a test to patients. In addition, informed consent to testing has been recommended in pretest guidelines from the World Health Organisation $^{1}$ and the $\mathrm{BMA},{ }^{2}$ which may intimidate doctors as there is no consensus about what constitutes informed consent. ${ }^{3}$ HIV and AIDS counselling is not psychotherapy and there is no right or wrong way to counsel in medical settings. Ultimately, the decision to test lies at the confluence of the doctor's clinical judgment and the patient's personal views and psychological circumstances. The overall aim of counselling before an HIV antibody test is to "provide individuals who are considering being tested with information on the technical aspects of screening and the possible personal, medical, social, psychological and legal implications of being diagnosed either HIV positive or negative" (WHO guidelines, p22'). Counselling also provides an opportunity to educate people about the risks of transmission and discuss behaviours that may reduce these risks. ${ }^{4}$

\section{POINTS TO CONSIDER}

Discussion about testing should be initiated by doctors when HIV infection is part of a differential diagnosis or because it is considered that the person may be at risk for HIV infection. Counselling about HIV infection may be easier if the following points are first considered.

Confidentiality is of prime importance to the patient. Before seeing the patient consider how a record of the discussion with the patient will be kept and how the result will be recorded. In general practice the negative consequences for the patient who later applies for life insurance and who has on record that he or she has had a test may deter some patients from being tested through their general practitioner. HIV testing at a sexually transmitted diseases clinic or other anonymous testing site may be preferred by the patient.

Privacy-Ensure that there is a measure of privacy as the contents of the conversation may be sensitive.

Time-Ensure that you have around 10-15 minutes of uninterrupted time with the patient. It is not possible to cover all the issues adequately in less time. Be prepared to see the patient again if necessary.

Sensitive issues-Some discussion about intimate sexual practices and terminal illness is usually part of talking to people about HIV and AIDS. If you are uncomfortable doing this it may help to convey this to the patient by saying, for example, "I need to ask you some very personal and sensitive questions. This is not easy for me." This effectively frees the conversation from any impasse and eliminates resistance between you and the patient.

Blame-Patients who receive a positive test result may be upset, and their responses may include anger and aggression. It is important to remember that some doctors find giving bad news difficult, and they may feel responsible for giving the bad news and causing emotional upset.

Continuing care-Ensure before testing that you can make a referral to a physician and counsellor in HIV and AIDS should ongoing care be needed for the patient.

\section{Pre-HIV test counselling}

Some specific points ought to be covered in pre-HIV test counselling in accordance with WHO and BMA guidelines. Feedback from patients suggests that they appreciate the opportunity for a frank discussion and some preparation for the worst, even if the procedure raises their anxiety.

\section{NEED FOR TESTING}

For those requesting the test identify the need for testing. The objective is to decide whether there is a genuine risk for HIV infection or whether the request for testing is based on an incorrect perception of riskfor example, sitting next to someone who may be infected with HIV. Some suggestions for opening the conversation with the patient are as follows:

"What made you decide to have a test?"

"Have you been tested previously? If so, when?"

Ask the patient, "Why has the idea about a test come up now?" Answers often disclose information about the patient's psychosocial state-for example, "I can't go on worrying"; "I'm starting a new relationship"; "I think my husband is having an affair."

"I have some thoughts about the symptoms you have described. Have you ever thought of having an HIV test?"

\section{IDENTIFYING RISK ACTIVITY}

Discuss what has put the person at risk for HIV infection. Identify the risk activity rather than the risk group of the patient in assessing the risk.

\section{Sexual}

"What have you done in the past to consider yourself to be at risk?"

"Can you tell me whether you have had unprotected 
intercourse in the past? If so, when was the last time? Was this with a man or a woman?"

Sex may mean different things to different people, so it is important to explore the range of potentially risky activities. "What do you mean by havin 'sex'? Do you mean cuddling, kissing, anal or vaginal intercourse? I need to have an understanding of the activity that you think was the source of risk in order to ascertain your risk."

If it was a sexual risk ask if the contact was from west Africa as this may suggest the need for an HIV-II test.

\section{Injecting drug use}

"Have you ever injected drugs?"

"Have you ever shared needles, syringes, or works? When was the last time?"

\section{Blood and blood products}

"Have you ever had a blood transfusion?"

"Was it before 1985?"

"Was it in Britain?"

"Have you received any blood products? Can you remember what they were?"

\section{OTHER INFORMATION ON HISTORY OF RISK}

Gather other information which relates to the history of the risk:

"Have you ever been treated for any sexually transmitted disease?"

"Do you have any symptoms or worries about your health?"

"What illnesses have you had in the past?"

\section{TIMING OF LAST RISK ACTIVITY}

Identify when the last risk activity for HIV infection occurred. The HIV antibody test is not a "morning after" test; it takes between six weeks and three months before antibodies may be detected in the blood. Patients may need to delay testing or arrange for retesting, and a few may need psychological support to cope with stress and anxiety during this time.

\section{PATIENT'S UNDERSTANDING OF TRANSMISSION}

Ensure the patient understands the transmission process.

Transmission-HIV is passed by infected semen, vaginal fluid, or blood entering the body via the anus, mouth, vagina, mucous membranes, or a break in the skin; sharing of needles, syringes, and sex toys; blood and blood products before donor screening and heat treatment of blood products in 1985; and from mother to baby, either in utero or during breast feeding.

No evidence of transmission-HIV is not passed by social contact and domestic events (for example, sharing towels or cutlery) or by donating blood.

NATURE OF HIV ANTIBODY TEST

Describe the HIV test:

"It is a blood test."

"It is not an AIDS test. It is a test for antibodies to the HIV virus. The test result will tell us whether or not you are infected. It will not indicate how long you have been infected or when you might develop AIDS. At present we think that it takes an average of 8-10 years from infection with the virus to the development of clinical problems."

\section{ADVANTAGES AND DISADVANTAGES OF BEING TESTED}

Discuss the advantages and disadvantages of being tested. (There is seldom time in 10-15 minutes to discuss all of these fully. In brief interviews the focus is on medical advantages, life insurance, and social disadvantages.)
Advantages - (a) Prophylaxis and treatment at an early stage may prolong life and alter the course of illness. Symptoms can be identified and treated promptly and often more effectively. (b) Knowing the result may reduce stress and depression, even for people who are HIV positive, as it ends the period of uncertainty.

Disadvantages - (a) Life insurance may be withheld or higher premium policies be refused for people who take the test, and almost certainly if they are HIV positive. (b) There is social stigma in being tested. It may lead to relationship difficulties (family and social) or problems with employment, housing, and international travel.

\section{HOW THE TEST IS DONE}

Explain the test procedure:

"A small sample of blood will be taken."

"The result will be available on ... at ...." (Avoid Friday afternoons or late appointments as support and referrals may be limited or difficult to arrange.)

"The result is either positive or negative. A positive result means that antibodies have been found in the blood and that you are infected with HIV. We always repeat positive tests to confirm this. A negative result means that antibodies were not detected and you are unlikely to be infected unless the risk activity has been in the past three months, in which case we will need to repeat the test."

"I will give you the result in person. I will do this straight away when we meet again so that we can use the remaining time to discuss what the result means for you."

\section{COPING WITH A POSITIVE RESULT}

Assess how the patient might cope with a positive test result:

"There has been a risk for HIV infection and that is why you are having a test. If the result comes back positive who might you tell? What support might there be for you? What might be your greatest concern? How do you see yourself coping?" (Open ended questions addressing practical concerns rather than "How would you feel?" questions help people to focus on what actions they might take to support themselves.)

\section{PROCEDURES FOR FOLLOW UP}

Describe the procedure for follow up care if the result is positive:

"An HIV test is limited in that it doesn't tell us anything about your general health or what effect the virus is having on your immune system. A positive result is for us the beginning of finding out about your health." (Introducing uncertainty helps to convey some hope to the patient.)

"There are other tests and investigations that can be done which can help us to plan your care and treatment if you are found to be positive." (List the procedures: follow up appointments; referral to a specialist in HIV for medical follow up including monitoring of immune function, counselling, and social support; phone numbers of voluntary national and local support organisations - for example, the Terrence Higgins Trust, Positively Women, and AIDS help lines.)

\section{WAITING FOR THE TEST RESULT}

Assess the patient's ability to cope until the test result is available. Suggesting to patients that stress is normal and to be expected may help them to cope on their own until the result is available. "I know from experience that people sometimes feel more anxious after they have had the blood test. How will you manage if you get very worried before the result is available?" 
AN INFORMED DECISION?

Assess whether the patient is making an informed decision. This assessment is based on the patient's answers to questions and level of understanding and mental state. A patient answering yes to "Do you understand everything we've discussed about the HIV test?" is no evidence for informed consent. It is useful to ask patients to highlight the important ideas they most remember from the conversation. ${ }^{5}$ In particular, they should mention (or be reminded) of the following: it is not a test for AIDS; the meaning of a positive or negative result; one advantage and one disadvantage of the test.

In some clinics, particularly in the USA, doctors require the patient to sign a consent form. In our HIV testing clinic we feel that verbal consent is sufficient.

\section{SAFER PRACTICES}

Discuss reducing the risk for HIV infection. Use the pretest counselling as an opportunity for one to one health education and to promote safer sex and drug injecting practices with patients. Information can be given about where they can obtain free condoms, clean injecting equipment, or hepatitis B vaccination (which may be recommended to some patients).

"Avoid unprotected penetrative intercourse (vaginal or anal). Use condoms, a diaphragm, or cap and preferably spermicidal cream. Use only clean needles if you inject drugs."

\section{TIME TO DECIDE}

Some patients need some time to think before having the test. These patients should be given another appointment a few days later, if necessary, or referred to a specialist HIV counsellor if appropriate. There is hardly ever a clinical setting in which an immediate decision for an HIV test is a life or death issue.

\section{Conclusion}

The procedure for obtaining consent for an HIV test opens up communication between doctors and their patients and paves the way for comprehensive medical and psychological care and support for those found to be positive.

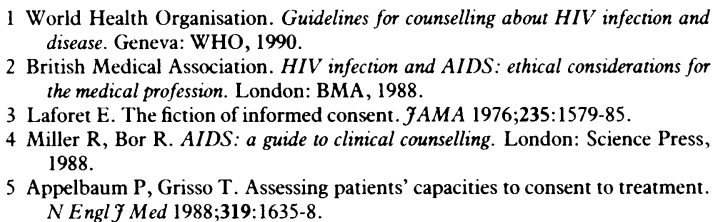

5 Appelbaum P, Grisso T. Assessing patients' capacities to consent to treatment. N Engl F Med 1988;319:1635-8.

(Accepted 12 fuly 1991)

\section{The New NHS: six months on}

\section{Budget holding: the first $\mathbf{1 5 0}$ days in Calverton}

\section{John Bain}

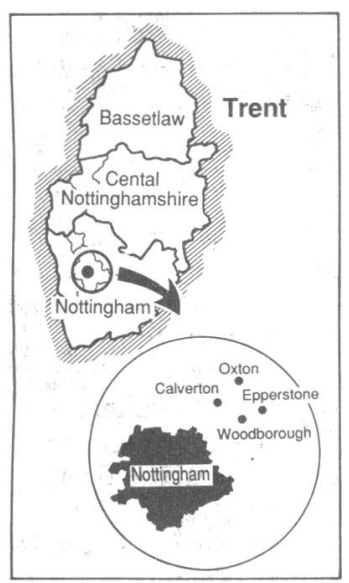

Levinson House,

Edinburgh EH8 9DX

John Bain, FRCGP, professor

of general practice

BMJ 1991;303:907-8 practice.
Disappointment with progress to date, yet not surprised with the way things are moving - that is the reaction of the Calverton practice as it reflects on the first three months of budget holding.

By holding a budget the partners in Calverton, a practice of five doctors based round three villages outside Nottingham, hoped to influence the behaviour of consultants with the end result being a reduction in waiting times for patients. How successful have they been so far in achieving this aim?

\section{Controlling hospital services}

Ground rules for outpatient and inpatient services are not yet established, and the current billing system is extremely cumbersome. The practice had drawn up specifications for minimum waiting times for patients and limits on the number of outpatient attendances, but no overall agreement has yet been reached about these proposals with the local hospitals. Allied to this the method of billing the practice is based on "paper exchanges" as the computer systems in the practice and in the hospital are not compatible.

According to the practice manager, "There are still a lot of bugs in the system," but he is still confident that these can be dealt with. The main frustration for him is that budget holding is up and running without an accountancy package which meets the needs of the

Plans to invite consultants to hold clinics in dermatology and rheumatology in the health centre have been held back. A compromise has been reached whereby a consultant dermatologist will see referred patients at a clinic in a private hospital. In time, this arrangement will be reviewed with the aim of placing the service within the Calverton health centre. In rheumatology a decision is still awaited about where the service for referred patients will be located. Reading between the lines I suspect that consultants may be encountering problems in providing services which satisfy both hospital managers and budget holding practices.

The original intention of the practice was not to go outside traditional providers of hospital care - that is, the NHS - but in the case of orthopaedics there are signs that the private sector will more readily be able to meet the practice's requirements in terms of waiting times and costs. Within the past three months arrangements have already been made for two patients waiting for hip replacements to have their operations performed in a private hospital. Although it is early days, the practice may increasingly have to go outside the NHS to reduce the waiting times for patients requiring surgical procedures.

Apart from laboratory services, there have been few attempts by providers in the private sector to woo the practice. But the practice has no plans at present to move away from NHS laboratory services.

\section{Changes in working habits}

Since the inception of budget holding there has been an estimated $10 \%$ reduction in the number of outpatient referrals and laboratory investigations, compared with a similar period in 1990 (April-June). The reasons for these changes have not been fully analysed, and the general practitioners are unwilling to reach any conclusions about trends in referral patterns until a longer period has elapsed. There have been no apparent alterations in drug prescribing behaviour, and plans for a drug formulary have been "put on ice" until there is enough time for the partners to get together and consider new policies on prescribing. 